Check for updates

\title{
Metallic iron in cornflakes $\uparrow$
}

\author{
Cite this: Food Funct., 2020, 11, 2938 \\ Joanna F. Collingwood, (D) ${ }^{a}$ Martin R. Lees, (D) ${ }^{c}$ Margaret P. Rayman (D) ${ }^{d}$ and \\ Peter J. Sadler (D) *b \\ Received 9th October 2019, \\ Accepted 13th February 2020 \\ DOI: $10.1039 /$ c9fo02370d \\ rsc.li/food-function

\begin{abstract}
Iron is an essential element, and cornflake-style cereals are typically fortified with iron to a level up to $14 \mathrm{mg}$ iron per $100 \mathrm{~g}$. Even single cornflakes exhibit magnetic behaviour. We extracted iron microparticles from samples of two own-brand supermarket cornflakes using a strong permanent magnet. Synchrotron iron $\mathrm{K}$-edge $\mathrm{X}$-ray absorption near-edge spectroscopic data were consistent with identification as metallic iron, and X-ray diffraction studies provided unequivocal identification of the extracted iron as bodycentred cubic (BCC) $\alpha$-iron. Magnetometry measurements were also consistent with ca. $14 \mathrm{mg}$ per $100 \mathrm{~g}$ $\mathrm{BCC}$ iron. These findings emphasise that attention must be paid to the speciation of trace elements, in relation to their bioavailability. To mimic conditions in the stomach, we suspended the iron extract in dilute $\mathrm{HCl}(\mathrm{pH} 1.0-2.0)$ at $310 \mathrm{~K}$ (body temperature) and found by ICP-MS that over a period of 5 hours, up to $13 \%$ of the iron dissolved. This implies that despite its metallic form in the cornflakes, the iron is potentially bioavailable for oxidation and absorption into the body.
\end{abstract}

\section{Introduction}

Of the 60 or so elements detectable in the human body, about 19 are known to be essential for human life, and a further seven are potentially essential. ${ }^{1-3}$ For various reasons, however, many individuals and population groups do not ingest appropriate amounts of all necessary elements through their regular diet. ${ }^{4-6}$ A common strategy to address this issue is to 'fortify' foods (i.e. supplement them) with these elements.

With nearly $5 \mathrm{~g}$ of iron present on average in the body, it is the most abundant essential transition metal for human health. ${ }^{1}$ The chemical form of iron in foods is known to influence its absorption in the body. For example, haem iron (e.g. in meat) is well absorbed, whereas non-haem iron in plantbased foods is less well absorbed as it can be bound in the gut by a number of dietary components, most notably phytate (myo-inositol hexakisphosphate), that inhibit iron absorption. ${ }^{7}$ Polyphenols, as found in tea, cereals and legumes have a similar effect. ${ }^{7}$ The most familiar form of iron in biology is in

\footnotetext{
${ }^{a}$ School of Engineering, University of Warwick, Coventry CV4 7AL, UK

${ }^{b}$ Department of Chemistry, University of Warwick, Coventry CV4 7AL, UK. E-mail: P.J.Sadler@warwick.ac.uk

${ }^{c}$ Department of Physics, University of Warwick, Coventry CV4 7AL, UK

${ }^{d}$ School of Biosciences and Medicine, Faculty of Health and Medical Sciences, University of Surrey, Guildford GU2 $7 X H$, UK

$\dagger$ Electronic supplementary information (ESI) available: Data for this paper can be accessed through the University of Warwick open access research repository (WRAP) at https://wrap.warwick.ac.uk/133560. See DOI: 10.1039/c9fo02370d

$\$$ These authors contributed equally.
}

haem groups; however, non-haem iron-containing proteins are encoded by approximately $1 \%$ of the human genome. ${ }^{1}$ The speciation of iron in the body after absorption is of much interest in relation to many diseases, including conditions of iron deficiency and overload, ${ }^{8}$ and neurodegenerative conditions such as Alzheimer's disease or neurodegeneration with brain iron accumulation. ${ }^{8-11}$

The presence of magnetic iron in cornflakes is well-known and its extraction using powerful permanent magnets is well described. ${ }^{12}$ Even a single cornflake exhibits magnetic behaviour (ESI video $\dagger$ ). We have analysed the iron extracted from samples of two own-brand UK supermarket cornflakes using synchrotron X-ray absorption near-edge spectroscopy (XANES), $\mathrm{X}$-ray diffraction, and magnetometry. We also investigated whether this iron might be bioavailable by studying its dissolution under temperature and $\mathrm{pH}$ conditions found in the stomach.

\section{Experimental}

\subsection{Iron extraction from cornflakes}

Two leading brands of cornflake (referred to as Brand A and Brand B), both reported by the manufacturers to contain $14.0 \mathrm{mg}$ of iron per $100 \mathrm{~g}$ of product - the highest iron level we were able to find among common breakfast cereals - were purchased from local (West Midlands, UK) grocery shops. Concentrated hydrochloric acid (37\%) was purchased from Sigma (Dorset, UK), and water was purified using a Milli-Q 
system. To extract iron-containing material, the cornflakes were ground by hand using a clean ceramic mortar and pestle, and then mixed with water and homogenised to a thin paste. This paste was then placed inside a glass beaker and stirred with a wooden stirrer, while a neodymium rare-earth magnet was placed against the outside wall of the beaker. After fifteen to thirty minutes of stirring, magnetic material was deposited as a dark pellet on the inside wall of the beaker. This material was then isolated and washed several times with Milli-Q water before being dried under air exposure at $310 \mathrm{~K}$ for further analysis.

\subsection{X-ray absorption spectroscopy}

Microfocus XANES experiments were performed at beamline I18 of the Diamond Light Source (Oxfordshire, UK). Careful tuning of the energy of the incident X-ray beam and scanning across a desired energy range, allows probing the oxidation state and local coordination environment of targeted elements within a sample. Particles extracted from cornflakes were suspended in Milli-Q water and $5 \mu \mathrm{L}$ of this suspension was deposited on a thin Ultralene film. This material was then allowed to dry for 2 hours at $310 \mathrm{~K}$ prior to XANES analysis.

\subsection{X-ray diffraction (XRD)}

Experiments were performed in transmission using a Xenocs Xeuss 2.0 equipped with a Mo K $\alpha$ microfocus source and a Pilatus $100 \mathrm{k}$ detector. The sample-to-detector distance was calibrated using a $\mathrm{LaB}_{6}$ standard. This setup allows the measurement of a diffraction pattern between 19 and $47^{\circ}$ in $2 \theta$. The extracted particles were mounted between Kapton tape which was also measured separately for background correction.

\subsection{Magnetometry}

DC magnetisation versus magnetic field measurements were made in a Quantum Design Magnetic Property Measurement System magnetometer, at temperatures between 5 and $300 \mathrm{~K}$ in magnetic fields of up to $50 \mathrm{kOe}$. Brand A cornflakes were crushed using a mortar and pestle and pressed into cylinders, $5 \mathrm{~mm}$ in height and $5 \mathrm{~mm}$ in diameter. Extracted iron samples were placed into gel capsules. The samples were then held in plastic straws which were attached to a sample rod for the measurements.

\subsection{Determination of solubilised Fe concentration by inductively coupled plasma - mass spectrometry (ICP-MS)}

Samples were diluted $(1: 2000)$ with $3.6 \%(\mathrm{v} / \mathrm{v})$ nitric acid and analysed using a 7500 Series ICP-MS instrument (Agilent Technologies, Palo Alto, CA, USA) in no-gas mode with ${ }^{166} \mathrm{Er}$ $\left(50 \mu \mathrm{g} \mathrm{L}^{-1}\right)$ as an internal standard. Calibration standards containing ${ }^{56} \mathrm{Fe}$ in $3.6 \%(\mathrm{v} / \mathrm{v})$ nitric acid from $0-500 \mu \mathrm{g} \mathrm{L}^{-1}$ were analysed in advance of sample analysis. To simulate conditions in the stomach, we prepared dilute aqueous $\mathrm{HCl}$ solutions at concentrations of $0.1,0.03$, and $0.01 \mathrm{M}$, corresponding to $\mathrm{pH}$ values of $1.0,1.5$, and 2.0 , respectively. These $\mathrm{pH}$ values are comparable to that of stomach acid, which typically has a
$\mathrm{pH}$ between 1.5 and $2.5{ }^{13,14}$ We then added $0.26-3.46 \mathrm{mg}$ of extracted iron particles to 0.5 or $1.0 \mathrm{~mL}$ of this diluted acid, and incubated the samples at $310 \mathrm{~K}$ for 5 hours (similar to the maximum reported residence time of food in the stomach). ${ }^{15,16}$ The sample was then filtered and the iron concentration in the supernatant determined by ICP-MS. Blanks consisting of Milli-Q water with $0,0.1,0.03$, and $0.01 \mathrm{M} \mathrm{HCl}$ with no extracted material from cornflakes were used as negative controls. Agilent ICP-MS Top and Offline Data Analysis (ChemStation version B.03.05) were used for data processing.

\section{Results and discussion}

\subsection{XANES spectroscopy revealed low-oxidation state iron}

As a first step, an optical image of the extracted particles was acquired. The inset in Fig. 1 shows dense, relatively smooth particles approximately $10 \mu \mathrm{m}$ in size. Microfocus XANES of these particles yielded a fairly noisy spectrum, possibly because of self-absorption, or potentially the particles were not fully immobilised on the substrate, while being of a comparable size to the incoming X-ray beam spot-size. Several peaks and a prominent shoulder ('pre-edge') at slightly lower photon energy than the main absorption edge were observed in the spectrum (Fig. 1) and were found at the same energy values as for a metallic iron standard. This strongly suggested that the

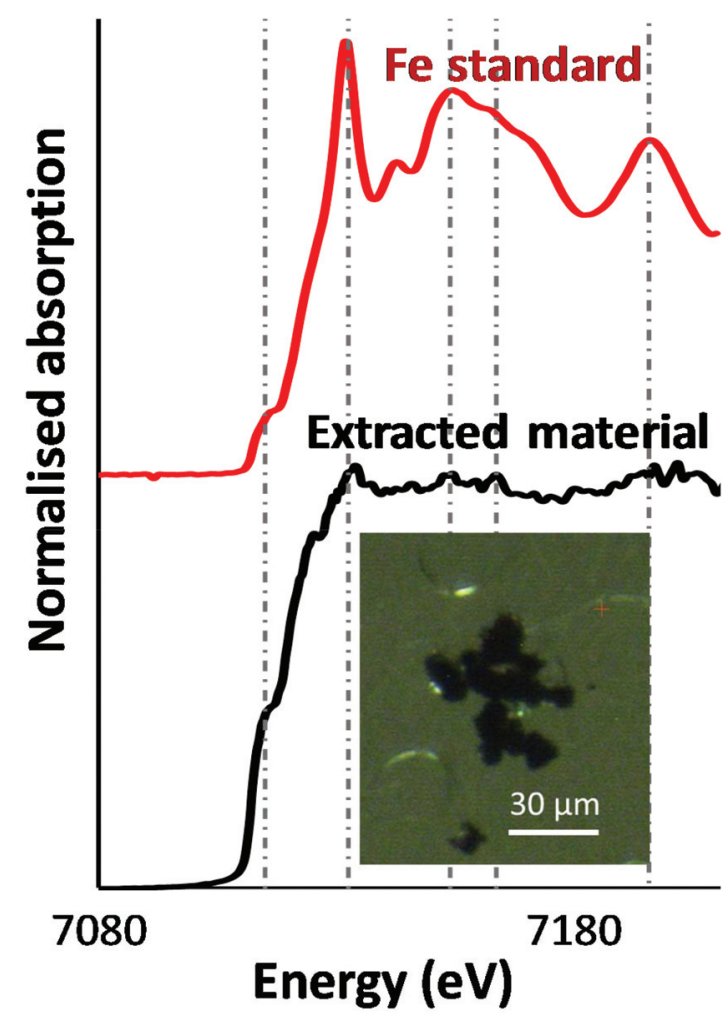

Fig. 1 XANES spectra of a pure, metallic iron standard (red trace) and of one of the ferromagnetic particles extracted from Brand A cornflakes (black trace). Dashed-dotted lines indicate matching features. Inset shows an optical image of the particles. 
iron in these particles was in zero-oxidation state, as higher oxidation states (especially +3 ) would cause the absorption maximum to shift to higher energy values. Given the noisy nature of the XANES spectrum, however, we performed a more detailed analysis of the particles using X-ray diffraction.

\subsection{XRD data were consistent with body-centred cubic alpha- iron}

Background-corrected X-ray diffraction data are plotted in Fig. 2 along with a simulated pattern for the body-centred cubic (BCC) $\alpha$-iron phase. ${ }^{17}$ The diffraction patterns of Brand A and Brand $\mathrm{B}$ are consistent with each other and match the expected pattern for BCC $\alpha$-iron. Together with the XANES data, this is extremely strong evidence that the extracted material consists of (metallic) $\alpha$-iron. We subsequently used magnetometry to measure the magnetic properties of the extracted iron.

\subsection{Magnetometry allowed determination of magnetisation and quantification of iron}

Fig. 3a shows the magnetization versus applied field curve at $295 \mathrm{~K}$ for a sample of Brand A cornflakes. The $M(H)$ curve is representative of all the samples of cornflakes investigated and consists of a ferromagnetic contribution from the iron and a diamagnetic contribution from the biological matter in the cornflakes. The cornflake residue after iron extraction exhibited a weaker diamagnetic response (see ESI Fig. S1-S3†). Fig. 3 b shows an $M(H)$ curve at $295 \mathrm{~K}$ for a sample of iron particles extracted from the cornflakes. As expected, the extracted iron was a soft ferromagnet, with a saturation magnetization, $M_{\text {sat }}=(196 \pm 1)$ emu $^{-1}$ at $50 \mathrm{kOe}$ and a coercive field of only $(8 \pm 2)$ Oe. The data for the extracted iron are compatible with previous results for BCC iron, and $M_{\text {sat }}$ is $\sim 90 \%$ of the 217.4 emu $\mathrm{g}^{-1}$ reported for bulk polycrystalline iron. ${ }^{18}$ The slightly reduced value may be due to the small size of the iron particles

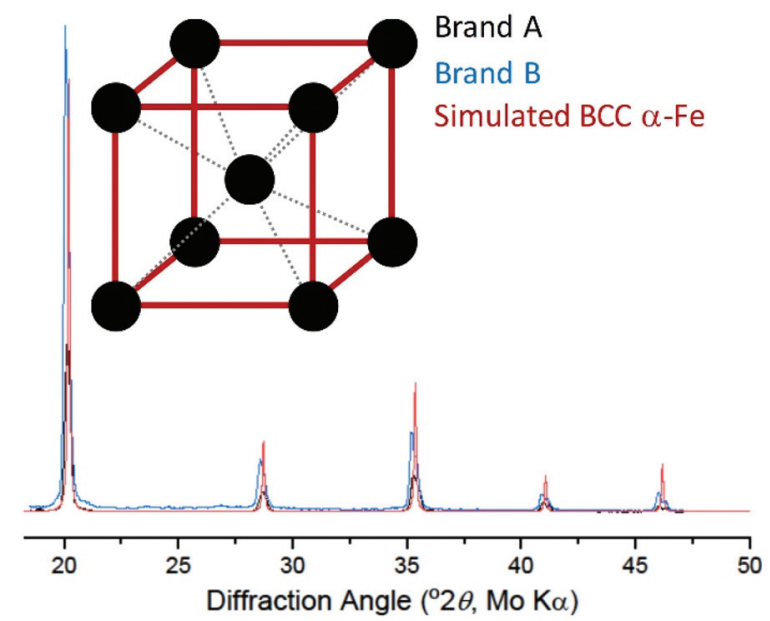

Fig. 2 X-ray diffraction of extracted cornflake particles (black, blue), along with simulated results for metallic body-centred cubic iron (red; structure shown in inset).
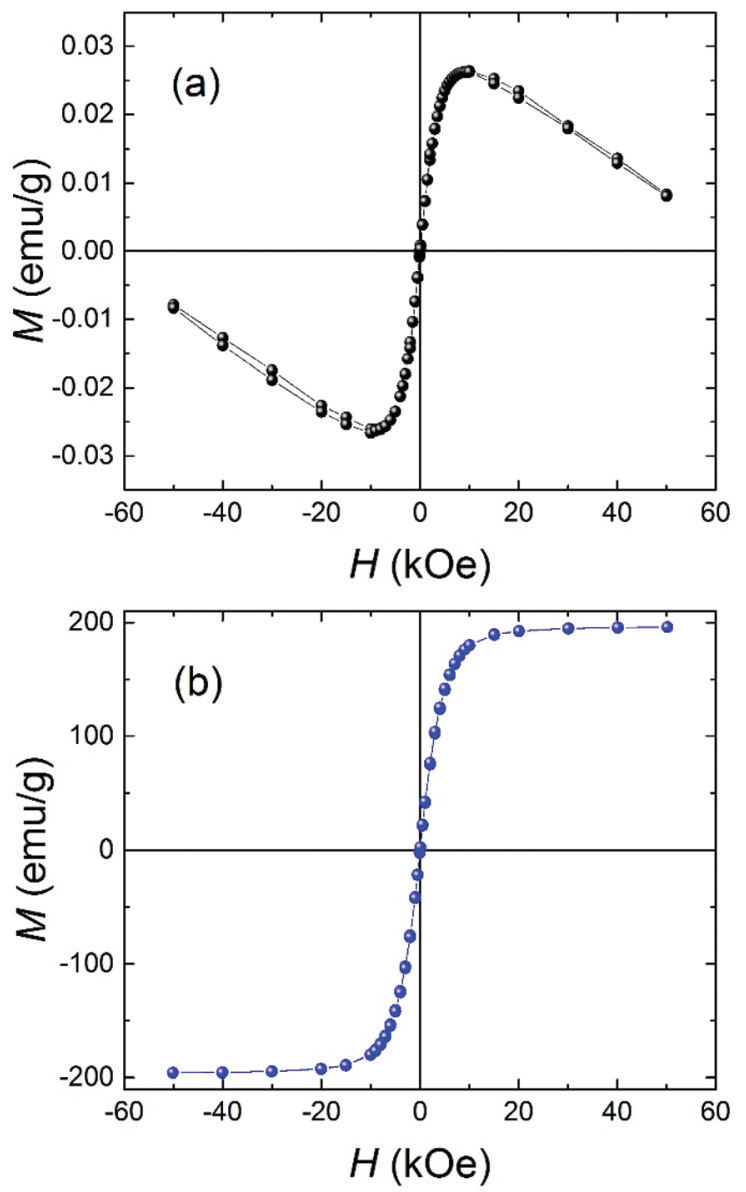

Fig. 3 Magnetization versus applied magnetic field at $T=295 \mathrm{~K}$ for (a) Brand A cornflakes and (b) the extracted iron particles.

and residual diamagnetic material in the extracted iron. Using the $M(H)$ curves, it is estimated the samples studied here contained $(12 \pm 1) \mathrm{mg}$ of iron per $100 \mathrm{~g}$ of cornflakes, in reasonable agreement with the $14 \mathrm{mg}$ stated by the manufacturer.

\subsection{The extracted iron slowly dissolved under physiological conditions}

It might be expected that little or none of the metallic iron particles in cornflakes would be available for absorption during digestion. However, it is well known that iron is readily oxidised and dissolves in aqueous acid, particularly at very low $\mathrm{pH}$ and high temperatures. We simulated conditions in the stomach as described in section 2.5, and determined the amount of solubilised iron by ICP-MS (Table 1). Strikingly, iron concentrations of 0.98 to $8.34 \mathrm{mM}$ were measured, suggesting that a significant fraction $(10.7 \pm 2.5 \%$ by mass $)$ of the iron particles was solubilised under conditions mimicking digestion. Generally, the reaction between metallic iron and hydrochloric acid under mild conditions generates iron(II) chloride, and the ferrous iron is soluble and relatively stable toward oxidation at low $\mathrm{pH} .{ }^{19}$ At $\mathrm{pH}$ values above 4 , the rate of oxidation to ferric iron by dissolved oxygen rapidly increases, 
Table 1 Summary of ICP-MS experiments. Background concentration of Fe measured in blank samples (Milli-Q $\mathrm{H}_{2} \mathrm{O}$ and acid at the three $\mathrm{pH}$ values used) was approximately $0.020 \mathrm{mM}$ and effectively negligible

\begin{tabular}{lllllr}
\hline & $\begin{array}{l}\text { Fe } \\
\text { particle } \\
\text { mass } \\
(\mathrm{mg})\end{array}$ & $\begin{array}{l}\text { HCl } \\
\text { volume } \\
(\mathrm{mL})\end{array}$ & $\begin{array}{l}\text { Fe } \\
\text { concentration } \\
(\mathrm{mM})\end{array}$ & $\begin{array}{l}\text { Amount of } \\
\text { Fe in acid } \\
(\mathrm{mg})\end{array}$ & $\begin{array}{l}\text { \% } \\
\text { dissolved }\end{array}$ \\
\hline 2.0 & 0.26 & 0.5 & 0.98 & 0.027 & 10.5 \\
1.5 & 2.53 & 1.0 & 3.85 & 0.215 & 8.5 \\
1.0 & 3.46 & 1.0 & 8.34 & 0.465 & 13.4 \\
& & & & & \\
\hline
\end{tabular}

and ferric iron is highly insoluble at neutral $\mathrm{pH} .{ }^{19}$ This raises the question as to whether the increase in $\mathrm{pH}$ when going from the stomach to the small intestine, might result in most of the dissolved iron precipitating as insoluble iron oxide clusters; however, binding by citrate or other chelators in the stomach could stabilise the iron sufficiently to be absorbed in the gut. ${ }^{20}$ In this context, we note again that chelating agents which might inhibit iron absorption such as phytate (inositol hexaphosphate) which is present not only in cereals, but also in bran, seeds, grains, legumes and many other plant tissues, can also have a significant detrimental effect on bioavailability. ${ }^{7,21}$ The membrane-bound ferric reductase Dcytb, found in the gut, has also been shown to play an important role in the absorption of dietary iron. ${ }^{20,22}$ This suggests that, after solubilisation in stomach acid, iron can plausibly be absorbed relatively efficiently despite having been ingested in a metallic form.

\section{Conclusions}

With increasing awareness of the importance of eating a balanced diet that contains all elements essential for life, there is incentive for manufacturers to fortify everyday foodstuffs. While the presence and concentration of trace elements in these items is well-known and, in many cases, reported, the chemical form of these elements is also important for health, although there is generally less awareness of this fact. Here, we have determined that the iron present in two leading brands of breakfast cereal in the UK, is in metallic form. By simulating conditions in the human stomach, we have also shown that it is plausible that a portion of this iron is likely to be solubilised during digestion, although questions remain concerning bioavailability. In particular, we emphasise that the model used here is highly simplified, and does not include consideration of factors such as the interaction of iron with components of foodstuffs, including the potential chelating agents they contain, $\mathrm{pH}$ changes in the stomach over time, or movement of materials during digestion. Rather, the focus here is on the detailed characterisation of the iron particles in fortified cereal, as well as a proof-of-principle experiment that shows that some of the iron can be solubilised under conditions related to those in the stomach. This work will therefore serve as the basis for more detailed future studies of the dissolution of these metallic iron particles and the subsequent absorption of iron. The work highlights the power of X-ray methods and magnetometry for analysis of iron in solid samples with minimal treatment, as well as the importance of chemical speciation in food science.

\section{Conflicts of interest}

There are no conflicts of interest to declare.

\section{Acknowledgements}

This work was supported by EPSRC grants EP/N033191/1 (F. L., J. F. C., P. J. S.), and EP/P030572/1 (P. J. S.), and by the University of Warwick through a Chancellor's Scholarship (for W.-Y. Z.). We thank Professor Richard I. Walton (Department of Chemistry, University of Warwick) for advice on the XRD experiments, and Dr Tina Geraki (Diamond Light Source) for assistance in acquiring XANES spectra. We are grateful to the reviewers for their valuable comments.

\section{References}

1 P. Chellan and P. J. Sadler, The elements of life and medicines, Philos. Trans. R. Soc., A, 2015, 373, 20140182.

2 M. A. Zoroddu, J. Aaseth, G. Crisponi, S. Medici, M. Peana and V. M. Nurchi, The essential metals for humans: a brief overview, J. Inorg. Biochem., 2019, 195, 120-129.

3 W. Maret, The Metals in the Biological Periodic System of the Elements: Concepts and Conjectures, Int. J. Mol. Sci., 2016, 17, 66.

4 M. P. Rayman, Selenium and human health, Lancet, 2012, 379, 1256-1268.

5 S. C. Bath and M. P. Rayman, Has the UK really become iodine sufficient?, Lancet Diabetes Endocrinol., 2018, 6, 8990.

6 S. C. Bath, C. D. Steer, J. Golding, P. Emmett and M. P. Rayman, Effect of inadequate iodine status in UK pregnant women on cognitive outcomes in their children: results from the Avon Longitudinal Study of Parents and Children (ALSPAC), Lancet, 2013, 382, 331-337.

7 R. Hurrell and I. Egli, Iron bioavailability and dietary reference values, Am. J. Clin. Nutr., 2010, 91, 1461S-1467S.

8 P. Brissot and O. Loreal, Iron metabolism and related genetic diseases: A cleared land, keeping mysteries, J. Hepatol., 2016, 64, 505-515.

9 J. Everett, J. F. Collingwood, V. Tjendana-Tjhin, J. Brooks, F. Lermyte, G. Plascencia-Villa, I. Hands-Portman, J. Dobson, G. Perry and N. D. Telling, Nanoscale synchrotron X-ray speciation of iron and calcium compounds in amyloid plaque cores from Alzheimer's disease subjects, Nanoscale, 2018, 10, 11782-11796.

10 N. D. Telling, J. Everett, J. F. Collingwood, J. Dobson, G. van der Laan, J. J. Gallagher, J. Wang and A. P. Hitchcock, Iron 
Biochemistry is Correlated with Amyloid Plaque Morphology in an Established Mouse Model of Alzheimer's Disease, Cell Chem. Biol., 2017, 24, 1205-1215.

11 J. F. Collingwood and M. R. Davidson, The role of iron in neurodegenerative disorders: insights and opportunities with synchrotron light, Front. Pharmacol., 2014, 5, 191.

12 E. K. Jacobsen and J. Maynard, Iron in Breakfast Cereal. Demonstrations for National Chemistry Week 2004, J. Chem. Educ., 2004, 81, 1544.

13 T. L. Russell, R. R. Berardi, J. L. Barnett, L. C. Dermentzoglou, K. M. Jarvenpaa, S. P. Schmaltz and J. B. Dressman, Upper gastrointestinal $\mathrm{pH}$ in seventy-nine healthy, elderly, North American men and women, Pharm. Res., 1993, 10, 187-196.

14 J. B. Dressman, R. R. Berardi, L. C. Dermentzoglou, T. L. Russell, S. P. Schmaltz, J. L. Barnett and K. M. Jarvenpaa, Upper gastrointestinal (GI) pH in young, healthy men and women, Pharm. Res., 1990, 7, 756761.

15 M. Camilleri, L. J. Colemont, S. F. Phillips, M. L. Brown, G. M. Thomforde, N. Chapman and A. R. Zinsmeister, Human gastric emptying and colonic filling of solids characterized by a new method, Am. J. Physiol., 1989, 257, G284-G290.
16 J. J. Bonner, P. Vajjah, K. Abduljalil, M. Jamei, A. RostamiHodjegan, G. T. Tucker and T. N. Johnson, Does age affect gastric emptying time? A model-based meta-analysis of data from premature neonates through to adults, Biopharm. Drug Dispos., 2015, 36, 245-257.

17 A. W. Hull, A New Method of X-Ray Crystal Analysis, Phys. Rev., 1917, 10, 661-696.

18 C. D. Graham, Iron and nickel as magnetization standards, J. Appl. Phys., 1982, 53, 2032-2034.

19 B. Morgan and O. Lahav, The effect of $\mathrm{pH}$ on the kinetics of spontaneous $\mathrm{Fe}(\mathrm{II})$ oxidation by $\mathrm{O}_{2}$ in aqueous solutionbasic principles and a simple heuristic description, Chemosphere, 2007, 68, 2080-2084.

20 E. C. Theil, Iron, ferritin, and nutrition, Annu. Rev. Nutr., 2004, 24, 327-343.

21 T. Hazell and I. T. Johnson, In vitro estimation of iron availability from a range of plant foods: influence of phytate, ascorbate and citrate, Br. J. Nutr., 1987, 57, 223-233.

22 A. T. McKie, D. Barrow, G. O. Latunde-Dada, A. Rolfs, G. Sager, E. Mudaly, M. Mudaly, C. Richardson, D. Barlow, A. Bomford, T. J. Peters, K. B. Raja, S. Shirali, M. A. Hediger, F. Farzaneh and R. J. Simpson, An iron-regulated ferric reductase associated with the absorption of dietary iron, Science, 2001, 291, 1755-1759. 